\title{
Upregulated miR-411-5p levels promote lymph node metastasis by targeting RYBP in head and neck squamous cell carcinoma
}

\author{
CHI ZHANG ${ }^{1,2^{*}}$, HONGFEI WANG ${ }^{1,2^{*}}$, MIAO DENG ${ }^{1,2}$, LIHONG HE $^{1,2}$, FAN PING $^{1,2}$,

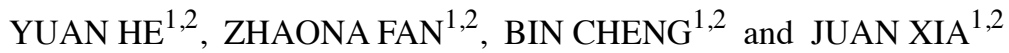 \\ ${ }^{1}$ Department of Oral Medicine, Hospital of Stomatology, Sun Yat-Sen University; ${ }^{2}$ Guangdong Provincial Key Laboratory of \\ Stomatology, Guanghua School of Stomatology, Sun Yat-Sen University, Guangzhou, Guangdong 510055, P.R. China
}

Received September 22, 2020; Accepted December 15, 2020

DOI: $10.3892 / \mathrm{ijmm} .2021 .4869$

\begin{abstract}
Metastasis is the primary cause of the high mortality rates in head and neck squamous cell carcinoma (HNSCC). MicroRNA (miR)-411-5p has been discovered to serve an important role in cancer metastases. However, to the best of our knowledge, the association between miR-411-5p expression levels and HNSCC metastasis has not been thoroughly investigated. The present study aimed to research the function of miR-411-5p in HNSCC metastasis. The results of the present study revealed that miR-411-5p expression levels were upregulated in patients with HNSCC with lymph node metastasis and the upregulated expression levels of miR-411-5p were positively associated with the metastatic potential of HNSCC. Moreover, miR-411-5p promoted HNSCC cell migration, invasion and epithelial-mesenchymal transition (EMT). The results of the dual-luciferase reporter assays identified RING1 and YY1 binding protein (RYBP) as a functional downstream target gene for miR-411-5p. Therefore, whether miR-411-5p downregulated the expression levels of RYBP in HNSCC cells was subsequently investigated. Notably, the silencing of RYBP expression restored the stimulatory effects of miR-411-5p on HNSCC cell migration, invasion and EMT. In addition, the mRNA expression levels of miR-411-5p and RYBP were found to be inversely correlated in HNSCC samples. In conclusion, the results of the present study indicated that the miR-411-5p-mediated downregulation of RYBP expression levels may exert an important role in HNSCC metastasis and may provide a novel target for the treatment of HNSCC.
\end{abstract}

Correspondence to: Professor Bin Cheng or Professor Juan Xia, Department of Oral Medicine, Hospital of Stomatology, Sun Yat-Sen University, 56 Lingyuan West Road, Guangzhou, Guangdong 510055, P.R. China

E-mail: chengbin@mail.sysu.edu.cn

E-mail: xiajuan@mail.sysu.edu.cn

*Contributed equally

Key words: head and neck squamous cell carcinoma, metastasis, epithelial mesenchymal transition, microRNA-411-5p, RING1 and YY1 binding protein

\section{Introduction}

Head and neck squamous cell carcinoma (HNSCC) is the sixth most common type of cancer worldwide and has a poor 5-year survival rate (1). According to statistics from the American Cancer Society, an estimated 53,000 new cases of HNSCC were diagnosed in the United States in 2019 (2) and globally, 600,000 new cases of HNSCC are diagnosed annually (1). Although there has been significant advancements in the diagnosis and treatment methods of HNSCC over the past few years, the 5-year survival rate for HNSCC has not significantly improved. Cervical lymph node metastasis (LNM) is an important and independent prognostic factor affecting the treatment of HNSCC, and is considered to be the main cause of the high mortality of HNSCC (3). Therefore, an improved understanding of the mechanisms of metastasis and the identification of novel biomarkers are urgently required to develop effective therapeutic strategies for HNSCC.

MicroRNAs (miRNAs/miRs) are a class of short non-coding RNAs of 18-24 nucleotides in length, which regulate gene expression by binding to the 3 -untranslated region (UTR) of target mRNAs to inhibit protein translation and/or degrade the mRNA $(4,5)$. An increasing number of studies have reported that miRNAs play important roles as oncogenes or tumor suppressor by regulating the biological behaviors of cancer cells (6-10). Moreover, accumulating studies have reported that numerous miRNAs are abnormally expressed in HNSCC tissues, which affects the cell migration and invasion of HNSCC (11-13).

miR-411 belongs to the $14 \mathrm{q} 32.31 \mathrm{miRNA}$ cluster (14). Previous studies have reported that miR-411-5p inhibits the metastasis of breast cancer cells (15) and the overexpression of miR-411 suppresses renal cell cancer migration (16). In non-small cell lung cancer (NSCLC), miR-411-5p/3p can promote NSCLC cell proliferation, tumor growth and metastasis both in vitro and in vivo (17). However, these studies mainly focused on the differences in miR-411-5p expression levels between cancer tissues and paracancerous tissues. To further understand the effect of miR-411-5p on metastasis in HNSCC, the present study investigated the differences in miR-411-5p expression levels between patients with and without LNM, and further analyzed the mechanism of action of miR-411-5p in regulating HNSCC metastasis. 
Ring1 and YY1 binding protein (RYBP) is a member of Polycomb group (PcG) proteins (18). It has been demonstrated that the dysregulation of RYBP leads to a poorer prognosis in hepatocellular carcinoma (19) and RYBP has been found to act as a tumor suppressor in different types of cancer (20-22). From previous research, it is known that different miRNAs can regulate RYBP expression in tumorigenesis in melanoma and gastric cancer $(23,24)$.

Thus, it was hypothesized that miR-411-5p can affect metastasis and epithelial-mesenchymal transition (EMT) by regulating RYBP expression in HNSCC cells. The present study investigated the effect of miR-411-5p on metastasis and EMT in vitro and in vivo, and further clarify the potential mechanisms of miR-411-5p in the development of HNSCC metastasis. The results of the present study may provide a novel prognostic and therapeutic strategy to treat HNSCC metastasis.

\section{Materials and methods}

The Cancer Genome Atlas (TCGA) analysis. The miRNA expression profiles of TGCA-HNSC dataset and the clinical information were obtained from TCGA database (https://portal. gdc.cancer.gov/) (25). A total of 528 patients with HNSCC from TCGA database were selected for analysis in the present study. The exclusion criteria were as follows: i) Incomplete information on LNM; ii) incomplete patient clinical data; and iii) the miR-411-5p expression data were incomplete. Following the exclusion of patients, the miR-411-5p expression levels and clinical information from 378 patients with HNSCC were used in the present study.

Patient studies. A total of 52 tissues from patients with HNSCC were collected from The Hospital of Stomatology, Sun Yat-Sen University (Guangzhou, China) between October 2017 and June 2018. No patient had received radiotherapy or chemotherapy before the biopsy, and all patients were diagnosed with HNSCC. The tissue samples were frozen at $-80^{\circ} \mathrm{C}$ until required for use in further experiments. The histology and pathology of all samples was examined by two independent pathologists. Written informed consent was provided from all patients prior to participation and the study was approved by the Ethics Committee of Sun Yat-Sen University [approval no. ERC-(2017)-32].

Cell lines and culture. The human HNSCC cell lines, HSC-3 and SCC-15, were purchased from the American Type Culture Collection. HSC-3 cells were cultured in DMEM supplemented with $10 \%$ FBS, $100 \mathrm{IU} / \mathrm{ml}$ penicillin and $100 \mu \mathrm{g} / \mathrm{ml}$ streptomycin. SCC- 15 cells were cultured in DMEM/F12 (1:1) supplemented with 10\% FBS, $100 \mathrm{IU} / \mathrm{ml}$ penicillin and $100 \mu \mathrm{g} / \mathrm{ml}$ streptomycin. All culture reagents were purchased from Gibco (Thermo Fisher Scientific, Inc.). All cells were maintained at $37^{\circ} \mathrm{C}$ in a humidified atmosphere with $5 \% \mathrm{CO}_{2}$.

Cell transfection and lentiviral vector construction. A total of $1 \times 10^{5}$ cells were transiently transfected with $50 \mathrm{nM}$ chemically synthesized miR-411-5p mimic, $100 \mathrm{nM}$ miR-411-5p inhibitor, $50 \mathrm{nM}$ small interfering RNA (si)-RYBP and the corresponding negative controls (NCs; all Guangzhou RiboBio Co., Ltd.) using Lipofectamine ${ }^{\circledR} 3000$ (Invitrogen; Thermo Fisher Scientific, Inc.), according to the manufacturer's protocols. The sequences were as follows: miR-411-5p mimic, 5'-UAGUAG ACCGUAUAGCGUACG-3'; miR-411-5p inhibitor, 5'-CGU ACGCUAUACGGUCUACUA-3'; and si-RYBP, 5'-ACAGCA TACAGTCTGCAAA-3'. Lentiviruses encoding a reverse complementary sequence of miR-411-5p (Anti-miR-411-5p) and an empty vector (Anti-ctrl) were constructed by Shanghai GeneChem Co., Ltd. A total of $1 \times 10^{5}$ cells HSC-3 and SCC-15 cells were infected with the lentiviral vectors $\left(1 \times 10^{8} \mathrm{TU} / \mathrm{ml}\right)$, and stable cell lines were selected following treatment with $2 \mu \mathrm{g} / \mathrm{ml}$ puromycin for 2 weeks.

$R N A$ extraction and reverse transcription-quantitative $P C R$ $(R T-q P C R)$. Total RNA was extracted from tissues or cultured cells using TRIzol ${ }^{\circledR}$ reagent (Invitrogen; Thermo Fisher Scientific,Inc.). To analyze the expression levels of miR-411-5p, Bulge-Loop ${ }^{\mathrm{TM}}$ miRNA qRT-PCR Primer sets were synthesized by Guangzhou RiboBio Co., Ltd. Total RNA was reverse transcribed into cDNA using the PrimeScript RT Master Mix (Takara Bio, Inc.), according to the manufacturer's protocols. qPCR was subsequently performed using the LightCycler ${ }^{\circledR}$ 480 SYBR Green I Master kit (Roche Diagnostics), according to the manufacturer's protocols. The following thermocycling conditions were used for qPCR: Initial denaturation at $95^{\circ} \mathrm{C}$ for $5 \mathrm{~min}$; followed by 40 cycles of denaturation for $10 \mathrm{sec}$ at $95^{\circ} \mathrm{C}$, annealing for $20 \mathrm{sec}$ at $60^{\circ} \mathrm{C}$ and extension for $20 \mathrm{sec}$ at $72^{\circ} \mathrm{C}$; and a final $10 \mathrm{~min}$ extension at $72^{\circ} \mathrm{C}$. The relative expression levels were calculated using the $2^{-\Delta \Delta C q}$ method (26). The following primers were used for the qPCR: RYBP forward, 5'-GGGGTGGTGGGGTGGCATACT-3' and reverse, 5'-CGC AGACGAAGGGTTTTGGGATT-3'; and GAPDH forward, 5'-GCACCGTCAAGGCTGAGAAC-3' and reverse, 5'-TGG TGAAGACGCCAGTGGA-3'. GAPDH and U6 were used as the internal controls for RYBP and miR-411-5p, respectively. GAPDH and RYBP were purchased from Sangon Biotech Co., Ltd., while U6 and miR-411-5p were purchased from Guangzhou RiboBio Co., Ltd.

Transwell assay. Cell migration and invasion assays were performed using Transwell chambers (8-mm pore size; Corning, Inc.). Briefly, for the migration assay, HSC-3 $\left(3 \times 10^{4}\right)$ or SCC-15 $\left(8 \times 10^{4}\right)$ cells were plated in serum-free medium into the upper chambers of the Transwell plates. For the invasion assay, HSC-3 $\left(6 \times 10^{4}\right)$ or SCC-15 $\left(2 \times 10^{5}\right)$ cells were seeded into the upper chambers with Transwell plates precoated with Matrigel for $6 \mathrm{~h}$ at $37^{\circ} \mathrm{C}$. The lower chambers were filled with medium supplemented with $10 \%$ FBS. Following incubation for $24 \mathrm{~h}$ at $37^{\circ} \mathrm{C}$, the medium was removed and the cells were fixed with $4 \%$ formaldehyde for $20 \mathrm{~min}$ at room temperature. Cells that had not migrated or invaded through the membrane were gently removed using a cotton swab. The fixed cells were subsequently stained with $0.4 \%$ crystal violet for $15 \mathrm{~min}$ at room temperature and observed under a light microscope (Zeiss $\mathrm{GmbH}$ ). A total of five representative fields at x100 magnification were randomly imaged. ImageJ v1.8.0 (National Institutes of Health) was used for quantification, and the average counts of the five images represented the migrated cells. 
Cell Counting Kit-8 (CCK-8) assays. The proliferation of HNSCC cells transfected with the miR-411-5p mimic or miR-411-5p inhibitor was determined using a CCK-8 assay (Telenbiotech). Briefly, $2 \times 10^{3}$ cells were seeded into a 96-well plate and cultured for $24 \mathrm{~h}$, prior to transfection with the miR-411-5p mimic or inhibitor. Following incubation for 24, $48,72,96$ or $120 \mathrm{~h}, \mathrm{CCK}-8$ reagent was added to the culture medium for $2 \mathrm{~h}$. The absorbance of each well was measured at a wavelength of $450 \mathrm{~nm}$. All experiments were performed in triplicate.

Colony formation assay. For the colony formation assay, $5 \times 10^{2}$ cells were seeded into 6 -well plates in triplicate $24 \mathrm{~h}$ post-transfection. Following the culture of cells for 10 days, the visible colonies were stained with $0.4 \%$ crystal violet for $10 \mathrm{~min}$ at room temperature. Colonies with diameters $>1 \mathrm{~mm}$ were defined as colonies.

Dual-luciferase reporter assay. To further analyze the molecular mechanism of miR-411-5p-mediated metastasis in HNSCC, miRDB (http://www.mirdb.org/miRDB), miRTarBase (http://mirtarbase.mbc.nctu.edu.tw/php/) and TargetScan 7.1 (http://www.targetscan.org/vert_71) databases were used. The potential miR-411-5p binding site in RYBP 3'-UTR was predicted using TargetScan (http://www.targetscan. org/vert_71). For luciferase assays, wild-type (Wt) or Mutant (Mut) RYBP 3'-UTR sequences were designed, synthesized and cloned into a pMIR-REPORT ${ }^{\mathrm{TM}}$ vector by $\mathrm{OBiO}$ Technology (Shanghai) Co., Ltd. to generate RYBP-3'-UTR-Wt and RYBP-3'-UTR-mut vectors, respectively. Then, HNSCC cells $\left(4 \times 10^{4}\right)$ were co-transfected with $100 \mathrm{nM}$ miR-411-5p mimics or NC and RYBP-3'-UTR-Wt or RYBP-3'-UTR-mut vectors using Lipofectamine 3000 reagent. Following $48 \mathrm{~h}$ of transfection, the relative luciferase activity was measured using a Dual-Luciferase Reporter Assay System (Promega Corporation), according to the manufacturer's protocols, and was compared with the Renilla luciferase activity of the samples. The assays were performed in triplicate.

Western blotting. Total protein was extracted from cells using RIPA lysis buffer (Beyotime Institute of Biotechnology) supplemented with protease and phosphatase inhibitors. Total protein was quantified using a BCA kit (Wuhan Boster Biological Technology Co., Ltd.), according to the manufacturer's protocols, and $20 \mu \mathrm{g}$ protein/lane was separated via SDS-PAGE on a $10 \%$ gel. The separated proteins were subsequently transferred onto PVDF membranes (EMD Millipore) and blocked with $5 \%$ non-fat milk for $1 \mathrm{~h}$ at room temperature. The membranes were then incubated with the following primary antibodies: Anti-E-cadherin (1:1,000; cat. no. 14472; Cell Signaling Technology, Inc.), anti-N-cadherin (1:1,000; cat. no. 13116; Cell Signaling Technology, Inc.), anti-vimentin (1:1,000; cat. no. 5741; Cell Signaling Technology, Inc.), anti-GAPDH (1:1,000; cat. no. 5174; Cell Signaling Technology, Inc.) and anti-RYBP (1:500; cat. no. sc-374235; Santa Cruz Biotechnology, Inc.). Following the primary antibody incubation, the membrane was washed three times with TBS with 2\% Tween-20 and incubated with HRP-conjugated anti-rabbit (cat. no. 7074) or anti-mouse (cat. no. 7076) secondary antibodies (1:5,000; Cell Signaling Technology, Inc.) for $1 \mathrm{~h}$ at room temperature. Protein bands were visualized using Immobilon Western Chemiluminescent HRP substrate (EMD

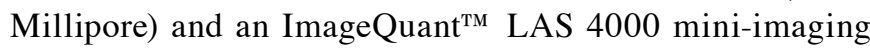
system (Cytiva). Semi-quantification was performed using Image J (v1.8.0).

Immunohistochemistry (IHC). IHC staining was performed with paraffin-embedded sections. Briefly, the lymph nodes were immediately fixed in $4 \%$ paraformaldehyde for $24 \mathrm{~h}$ and soaked in ethanol (70 to $100 \%$ ), following which the sections ( $4 \mu \mathrm{m}$ thick) were embedded in paraffin. The tissue sections were incubated in $10 \%$ goat serum blocking solution (Wuhan Boster Biological Technology, Ltd.) for $1 \mathrm{~h}$ at room temperature, followed by incubation with an anti-pan-cytokeratin primary antibody (1:200; cat. no. ab9377; Abcam) overnight at $4^{\circ} \mathrm{C}$. Sections were then incubated with a secondary antibody for $30 \mathrm{~min}$ at room temperature according to the instructions of the GT Vision ${ }^{\mathrm{TM}}$ and Detection System/MoRb kit (Gene Tech), which contained the secondary antibody and DAB, followed by staining with DAB and counterstaining with hematoxylin for $1 \mathrm{~min}$ at room temperature. Then, two senior pathologists blinded to the data assessed and scored the IHC results. The extent of staining was scored according to the percentage of positively stained cells: i) $1,<10 \%$ of cells; ii) $2,10-35 \%$ of cells; iii) $3,35-70 \%$ of cells; and iv) $4,>70 \%$ of cells. The staining intensity was graded as follows: i) 0 , no staining; ii) 1, weak staining (light yellow); iii) 2, moderate staining (yellow brown); and iv) 3, strong staining (brown), as previously described (9). The staining index was calculated by multiplying the percentage of positive cells and staining intensity. Five random fields per sample were analyzed using a light microscope (magnification, x100) to visualize sections.

In vivo xenograft experiments. All animal experiments were approved by the Ethical Committee of Sun Yat-Sen University (approval no. SYSU-IACUC-2020-000562) and were performed in accordance with the guidelines for animal care and protection (27). A total of $14 \mathrm{BALB} / \mathrm{c}$ nude mice (female; age, 4-6 weeks old; weight, 18-20 g) were obtained from GemPharmatech Co., Ltd., and were housed in specific pathogen-free conditions at $25^{\circ} \mathrm{C}$ and $50 \%$ humidity, with a 12-h light/dark cycle and free access to food and water. The mice were randomly divided into 2 groups $(n=7)$. A total of $2 \times 10^{6}$ SCC- 15 cells with or without miR-411-5p expression knockdown were suspended in $100 \mu \mathrm{l}$ PBS, and then injected into the tongue of the nude mice following anesthesia by isoflurane [ $4 \%(0.51 / \mathrm{min})$ for induction and $2 \%$ for maintenance]. Following 8 weeks, the maximum tumor diameter in the mice was recorded as $5.65 \mathrm{~mm}$ and the maximum tumor volume was $62.40 \mathrm{~mm}^{3}$, all mice were sacrificed using cervical dislocation and death was confirmed by the absence of breathing, and the lack of a heartbeat and corneal reflex. Finally, the cervical lymph nodes were collected and analyzed using IHC analysis.

Statistical analysis. Statistical analysis was performed using SPSS version 20.0 software (IBM Corp.) and GraphPad Prism 6.0 software (GraphPad Software, Inc.). Statistical differences between two groups were determined using an unpaired Student's t-test, while multiple group comparisons were performed using a one-way ANOVA, followed by 
Table I. Association between the expression of miR-411-5p and clinicopathological characteristics in HNSCC clinical sample.

\begin{tabular}{|c|c|c|c|c|}
\hline \multirow[b]{2}{*}{ Clinicopathological characteristics } & \multirow[b]{2}{*}{ Number of cases, $n$} & \multicolumn{2}{|c|}{ miR-411-5p expression, $n$} & \multirow[b]{2}{*}{ P-value } \\
\hline & & High & Low & \\
\hline \multicolumn{5}{|l|}{ Age, years } \\
\hline$\geq 55$ & 26 & 11 & 15 & \multirow[t]{2}{*}{0.406} \\
\hline$<55$ & 26 & 15 & 11 & \\
\hline \multicolumn{5}{|l|}{ Sex } \\
\hline Male & 37 & 17 & 20 & \multirow[t]{2}{*}{0.541} \\
\hline Female & 15 & 9 & 6 & \\
\hline \multicolumn{5}{|l|}{ Differentiation } \\
\hline Well & 23 & 7 & 16 & \multirow[t]{2}{*}{$0.025^{\mathrm{a}}$} \\
\hline Moderate + poor & 29 & 19 & 10 & \\
\hline \multicolumn{5}{|l|}{ T stage } \\
\hline T1 + TII & 26 & 7 & 14 & \multirow[t]{2}{*}{0.404} \\
\hline TIII + TIV & 19 & 16 & 8 & \\
\hline \multicolumn{5}{|l|}{ Clinical stage } \\
\hline I-II & 20 & 6 & 14 & \multirow[t]{2}{*}{$0.045^{\mathrm{a}}$} \\
\hline III-IV & 32 & 20 & 12 & \\
\hline \multicolumn{5}{|l|}{ LNM } \\
\hline LNM(-) & 25 & 7 & 18 & \multirow[t]{2}{*}{$0.005^{\mathrm{a}}$} \\
\hline $\mathrm{LNM}(+)$ & 27 & 19 & 8 & \\
\hline
\end{tabular}

${ }^{a} \mathrm{P}<0.05$. miR, microRNA; HNSCC, head and neck squamous cell carcinoma; LNM, lymph node metastasis; $\mathrm{T}$, tumor.

a Tukey's post hoc test. $\chi^{2}$ tests were used to determine the association between miR-411-5p expression levels and clinicopathological features. Spearman's correlation analyses were used to determine the correlation between miR-411-5p and RYBP expression. The Kaplan-Meier method and a log-rank test were used for overall survival analysis. To further determine the influence of multiple independent prognostic factors, including miR-411-5p expression levels, on the overall survival of patients with HNSCC, multivariate Cox regression analysis was performed. $\mathrm{P}<0.05$ was considered to indicate a statistically significant difference.

\section{Results}

miR-411-5p expression levels are upregulated in patients with HNSCC with LNM. In the present study, 378 patients with HNSCC from TCGA were obtained, including 218 patients with LNM and 160 patients without LNM. As shown in Fig. 1A, the expression levels of miR-411-5p were upregulated in patients with HNSCC with LNM compared with patients without LNM. To validate the results obtained via bioinformatics analysis, miR-411-5p expression levels in 52 HNSCC clinical patient samples were analyzed by RT-qPCR, and the results revealed the same trends in the expression levels as the bioinformatics analysis (Fig. 1B). These findings suggested that the expression levels of miR-411-5p may be associated with LNM in HNSCC.

Association between miR-411-5p expression levels and clinicopathological characteristics in patients with HNSCC.
As shown in Table I, $\chi^{2}$ analysis revealed that high miR-411-5p expression levels were positively associated with LNM, pathological differentiation and clinical stage in 52 clinical HNSCC patient samples. Furthermore, the association between miRNA-411-5p expression levels and the clinicopathological characteristics of the 378 patients with HNSCC are shown in Table SI. The analysis identified that miR-411-5p expression levels were associated with LNM; however, no association was found between miR-411-5p expression levels and sex, age, $\mathrm{T}$ stage, clinical stage and pathological differentiation. By combining the analysis of TCGA databases and HNSCC clinical samples, the data indicated that miR-411-5p expression levels may be a useful biomarker for HNSCC.

Association between miR-411-5p expression levels and prognosis in patients with HNSCC. Kaplan-Meier survival analysis based on TCGA dataset revealed that upregulated miR-411-5p expression levels were associated with a poor prognosis in patients with HNSCC (Fig. 2A). In addition, the prognostic value of miR-411-5p expression levels was also analyzed in patients with LNM. The results revealed that upregulated miR-411-5p expression levels were associated with a poor overall survival rate in patients with HNSCC with LNM (Fig. 2B). Furthermore, univariate analysis revealed that LNM and miR-411-5p expression levels were prognostic factors for patients with HNSCC. Multivariate cox analysis of the prognostic variables in 378 patients with HNSCC from TCGA dataset identified that miR-411-5p expression levels [hazard ratio (HR), 1.478; 95\% CI, 1.066-2.048; $\mathrm{P}=0.019$ ] and 
A

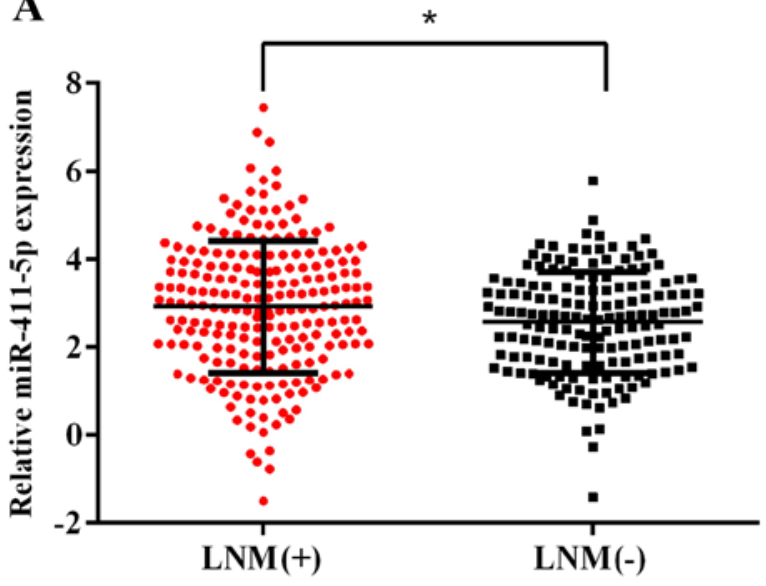

B

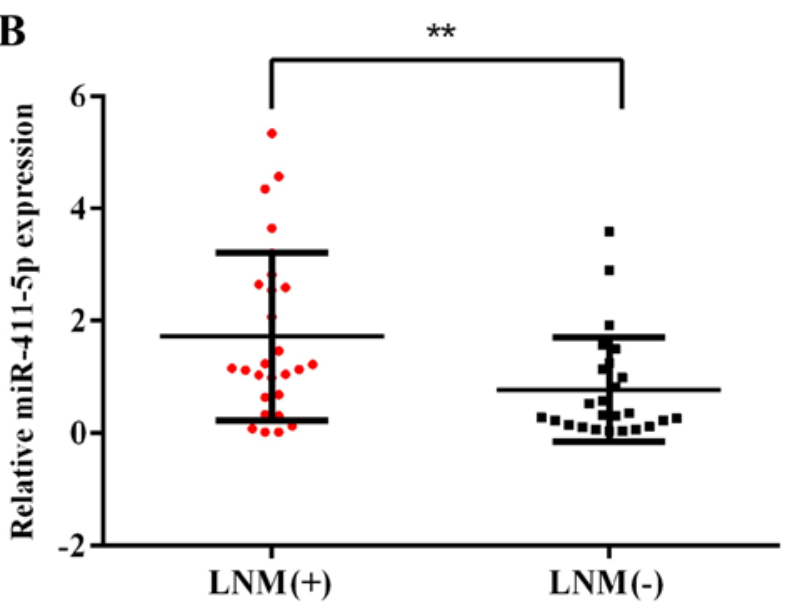

Figure 1. miR-411-5p expression levels are upregulated in patients with HNSCC with LNM. (A) Expression levels of miR-411-5p in patients with HNSCC with or without LNM from The Cancer Genome Atlas database. (B) Expression levels of miR-411-5p in patients with HNSCC with or without LNM were analyzed using reverse transcription-quantitative PCR. LNM(+), patients with LNM; LNM(-), patients without LNM. ${ }^{*}<0.05$, ${ }^{* *} \mathrm{P}<0.01$. miR, microRNA; HNSCC, head and neck squamous cell carcinoma; LNM, lymph node metastasis.

A

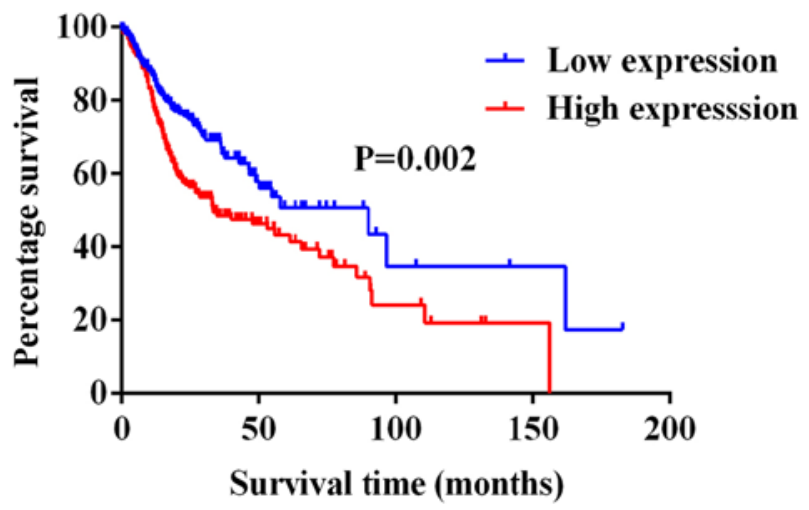

B

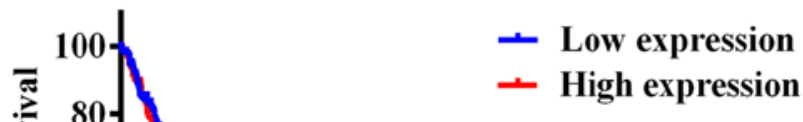

$\mathrm{P}=\mathbf{0 . 0 2 3}$

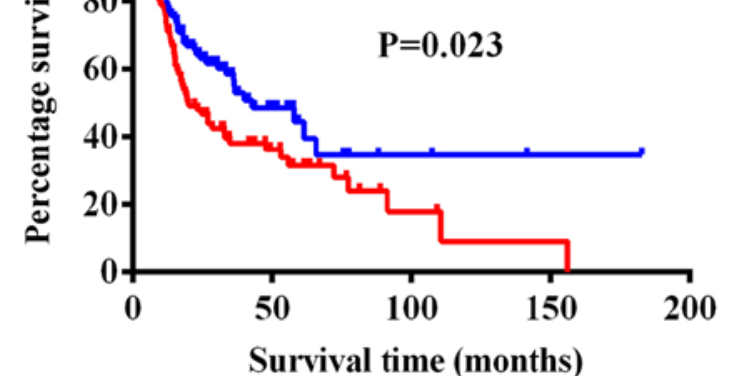

Survival time (months)

Figure 2. Upregulation of miR-411-5p expression levels is an indicator of an unfavorable prognosis in HNSCC. (A) Upregulated expression levels of miR-411-5p were associated with poor survival in patients with HNSCC from TCGA database. (B) Upregulated expression levels of miR-411-5p were associated with poor survival in patients with HNSCC with LNM from TCGA database. miR, microRNA; HNSCC, head and neck squamous cell carcinoma; LNM, lymph node metastasis; TCGA, The Cancer Genome Atlas.

LNM (HR, 1.911; 95\% CI, 1.335-2.735; P<0.001) were significantly associated with the prognosis of HNSCC (Table SII). Taken together, these results suggested that the upregulated expression levels of miR-411-5p may be positively associated with the metastatic potential of HNSCC and may serve as a potential indicator for the poor prognosis of patients with HNSCC.

miR-411-5p promotes HNSCC metastasis and EMT. Clinicopathological analyses demonstrated that miR-411-5p expression levels were positively associated with LNM in HNSCC. Therefore, the effects of miR-411-5p on the migration and invasion of HNSCC cells were analyzed using Transwell assays. HSC-3 and SCC-15 cells were transfected with miR-411-5p mimics or inhibitors, compared with the negative control group, increased and decreased miR-411-5p expression levels in HNSCC cells was confirmed by RT-qPCR (Fig. 3A and B). Meanwhile, HSC-3 and SCC15 cells were infected with lentiviral vectors to establish stable cell lines that expressed short hairpin RNA against miR-411-5p
(anti-miR-411-5p), decreased miR-411-5p expression was verified by RT-qPCR (Fig. S1A and B). In the miR-411-5p inhibitor group, the knockdown of miR-411-5p expression significantly reduced the migratory and invasive abilities of HSC-3 and SCC-15 cells (Fig. 3C). Conversely, the overexpression of miR-411-5p significantly promoted the migration and invasion of HSC-3 and SCC-15 cells (Fig. 3C). Furthermore, inguinal LNM models were constructed by injecting SCC-15 cells with or without knocked down miR-411-5p expression into the tongue of mice to analyze the effect of miR-411-5p on metastasis in vivo. Histological analysis revealed that the knockdown of miR-411-5p decreased the number of pan-cytokeratin-positive metastatic tumor cells and metastatic ratio of cervical lymph nodes (Fig. 3D and E). Taken together, these findings indicated that the knockdown of miR-411-5p may suppress LNM in vitro and in vivo.

It is well-established that the induction of EMT is a major event that promotes the motility of cancer cells in the invasion-metastasis cascade. Therefore, the effect of miR-411-5p on EMT was determined by analyzing the expression levels 
A

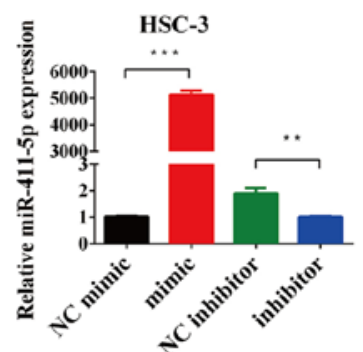

C

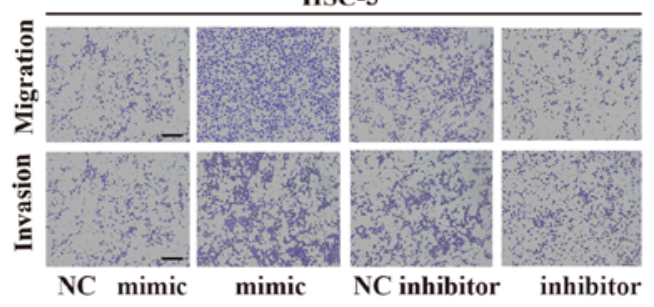

B
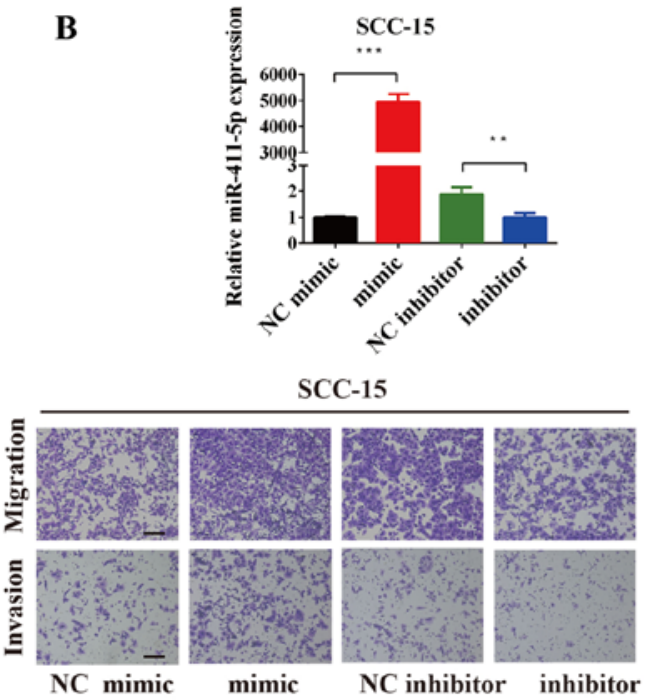

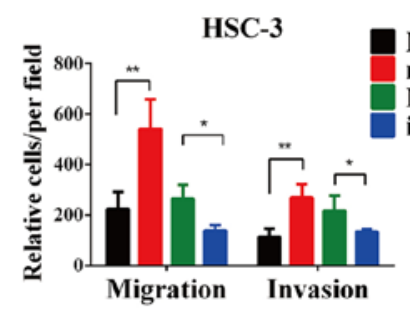

NC mimic

mimic

NC inhibitor

inhibitor

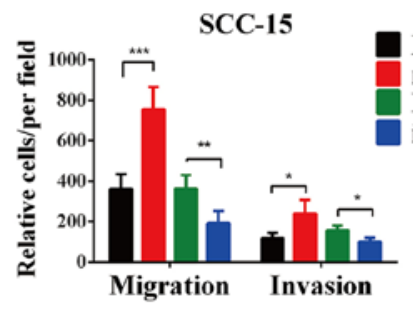

D

Anti-ctrl Anti-miR-411-5p

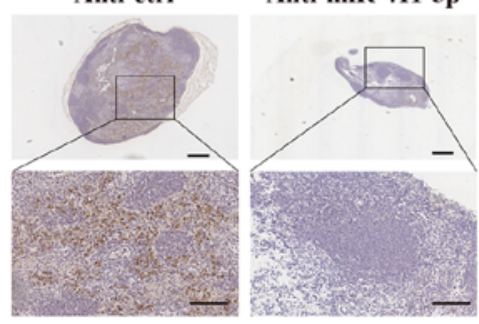

$\mathbf{E}$

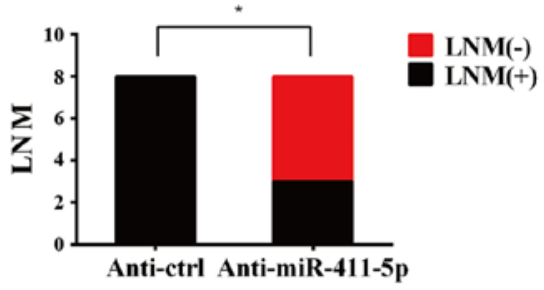

$\mathbf{F}$

HSC-3
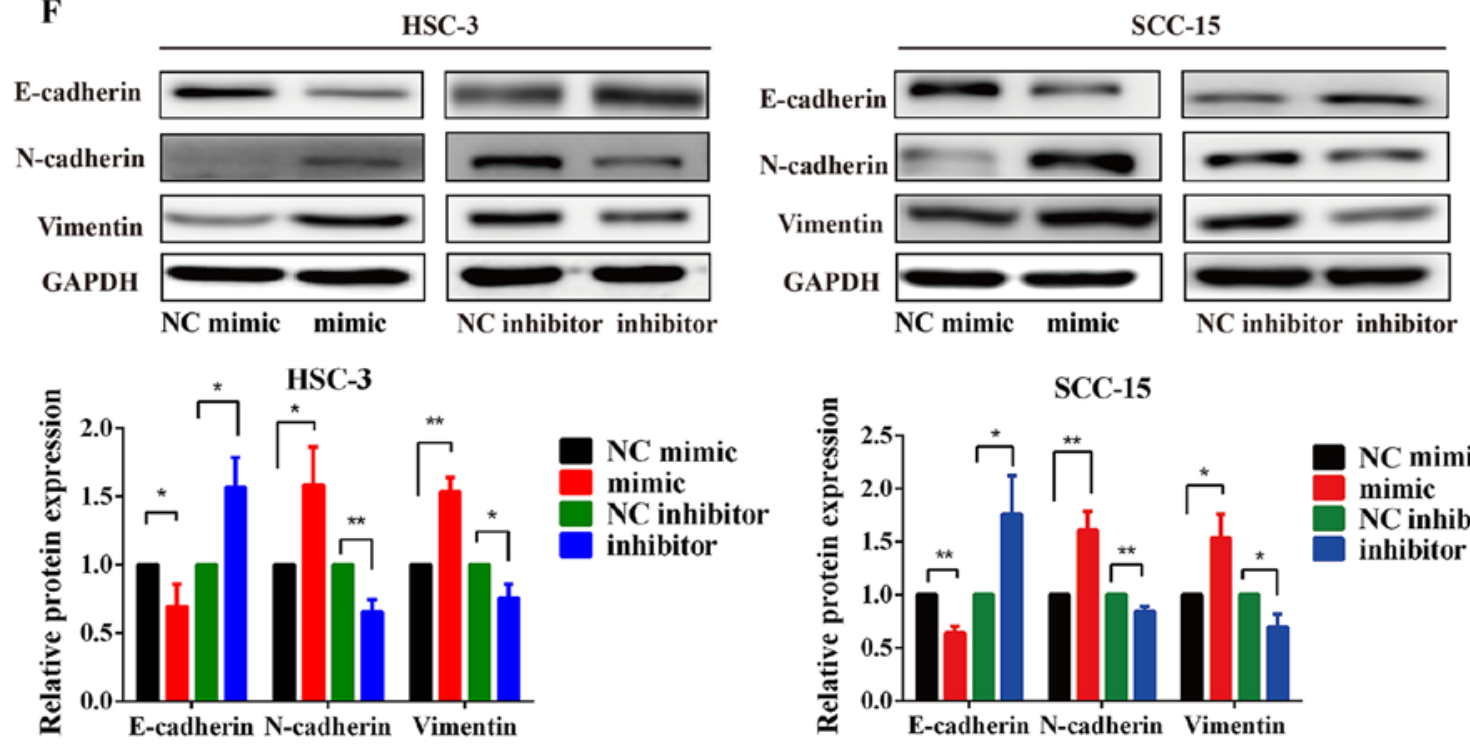

NC mimic mimic NC inhibitor inhibitor

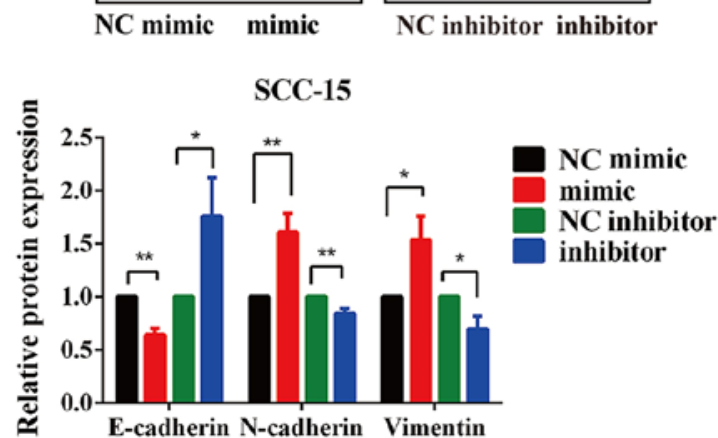

NC mimic

NC inhibitor

inhibitor

Figure 3. miR-411-5p promotes HNSCC cell migration, invasion and EMT in vitro and metastasis in vivo. (A and B) Transfection efficiency of miR-411-5p was verified using reverse transcription-quantitative PCR. (C) Effects of miR-411-5p mimics and inhibitors on the invasion and migration of HNSCC cells were analyzed using Transwell assays. (D) Representative staining of metastatic tumor cells in the cervical lymph nodes of mice following the injection of $2 \times 10^{6}$ SCC-15 cells with or without miR-411-5p inhibitor using anti-pan-cytokeratin. (E) Metastatic ratio of cervical lymph nodes. (F) Overexpression of miR-411-5p downregulated the expression levels of E-cadherin, and upregulated N-cadherin and vimentin expression levels in HNSCC cells. By contrast, the knockdown of miR-411-5p upregulated the expression levels of E-cadherin, and downregulated vimentin and N-cadherin expression levels. The expression levels of the EMT-related markers, E-cadherin, N-cadherin and vimentin were analyzed using western blotting. GAPDH was used as the internal loading control. Scale bar, $100 \mu \mathrm{m} .{ }^{*} \mathrm{P}<0.05,{ }^{* *} \mathrm{P}<0.01,{ }^{* * *} \mathrm{P}<0.001$. miR, microRNA; HNSCC, head and neck squamous cell carcinoma; EMT, epithelial-mesenchymal transition; NC, negative control; LNM, lymph node metastasis. 
A

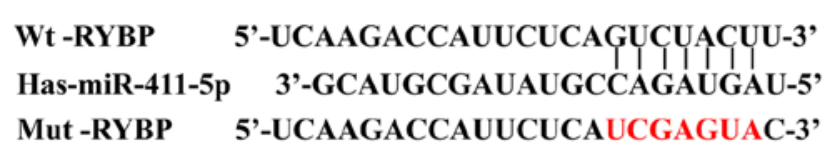

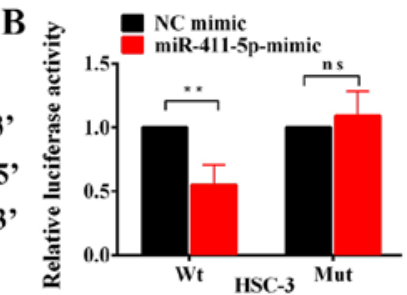

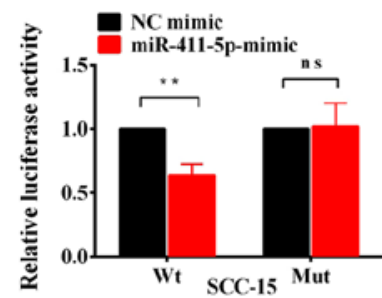

C
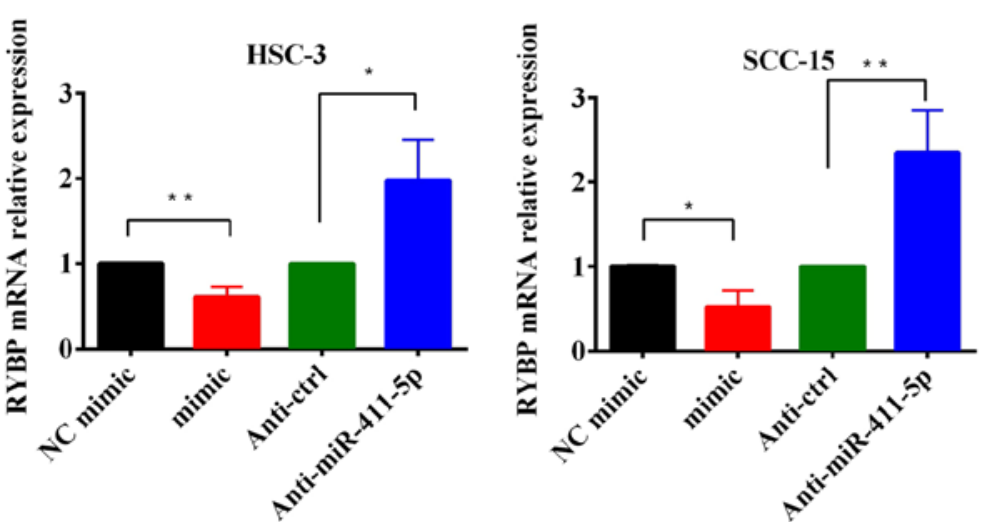

$\mathbf{E}$

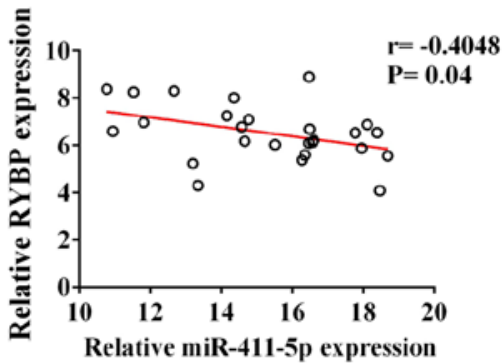

D

HSC-3
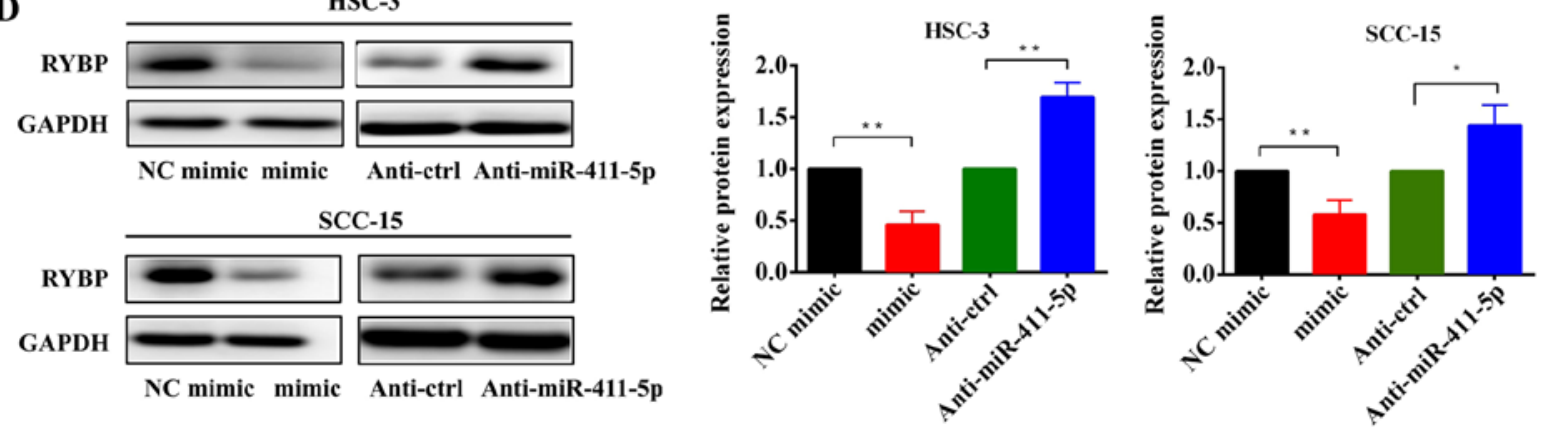

Figure 4. RYBP is a direct target gene of miR-411-5p in HNSCC cells. (A) Predicted and mut binding sites of miR-411-5p in the 3'-untranslated region of RYBP. (B) Relative luciferase activity was analyzed in HSC-3 and SCC-15 cells transfected with miR-411-5p mimic or NC and Wt or Mut reporter genes. (C and D) Reverse transcription-quantitative PCR and western blotting of RYBP mRNA and protein expression levels, respectively, following the overexpression and knockdown of miR-411-5p in HNSCC cells. (E) Spearman's correlation analysis was used to determine the correlation between RYBP and miR-411-5p expression levels in HNSCC tissues. "P<0.05, ${ }^{* *} \mathrm{P}<0.01$. RYBP, RING1 and YY1 binding protein; miR, microRNA; Wt, wild-type; Mut, mutant; NC, negative control; HNSCC, head and neck squamous cell carcinoma; ns, not significant.

of EMT-related markers. The overexpression of miR-411-5p downregulated the expression levels of the epithelial marker, E-cadherin, and upregulated the expression levels of the mesenchymal markers, N-cadherin and vimentin, in HNSCC cells (Fig. 3F). By contrast, the genetic knockdown of miR-411-5p upregulated the expression levels of $\mathrm{N}$-cadherin and vimentin and downregulated E-cadherin expression levels (Fig. 3F). These results indicated that miR-411-5p may promote the migration, invasion and EMT of HNSCC cells. Furthermore, CCK-8 and colony formation assays were conducted to analyze the proliferation of HNSCC cells. The results revealed that the overexpression and knockdown of miR-411-5p did not affect the proliferation of HNSCC cells (Fig. S2A and B).

$R Y B P$ is a direct target gene of miR-411-5p in HNSCC. The bioinformatics analysis demonstrated that the seed sequence of miR-411-5p was complementary to the 3'-UTR of RYBP (Fig. 4A), which suggested that RYBP may be a potential target of miR-411-5p. Dual-luciferase reporter assays confirmed that miR-411-5p overexpression significantly suppressed the relative luciferase activity of the RYBP-3'-UTR-Wt reporter gene, but had no effect on the RYBP-3'-UTR-Mut reporter gene (Fig. 4B). Moreover, RT-qPCR and western blotting confirmed that the overexpression of miR-411-5p significantly downregulated RYBP expression levels, while the silencing of miR-411-5p expression upregulated RYBP expression levels (Fig. 4C and D). Clinically, a negative correlation was identified between miR-411-5p and RYBP expression levels in HNSCC tissues (Fig. 4E). Collectively, these results suggested that RYBP may be a direct downstream target of miR-411-5p in HNSCC cells.

Knockdown of RYBP promotes HNSCC cell migration, invasion and EMT. As expected, the expression levels of RYBP were downregulated in patients with HNSCC with LNM compared with patients without LNM (Fig. 5A). To analyze the effects of RYBP on HNSCC cells, si-RYBP was transfected into HSC-3 and SCC-15 cells. The genetic knockdown of RYBP resulted in the significant downregulation of RYBP protein expression levels (Fig. 5B). The results of the Transwell assays showed that the knockdown of RYBP significantly promoted HNSCC cell migration and invasion compared with the 


\section{“}

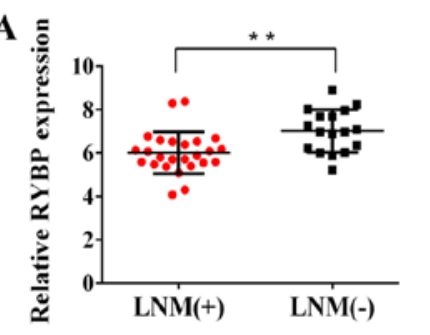

B
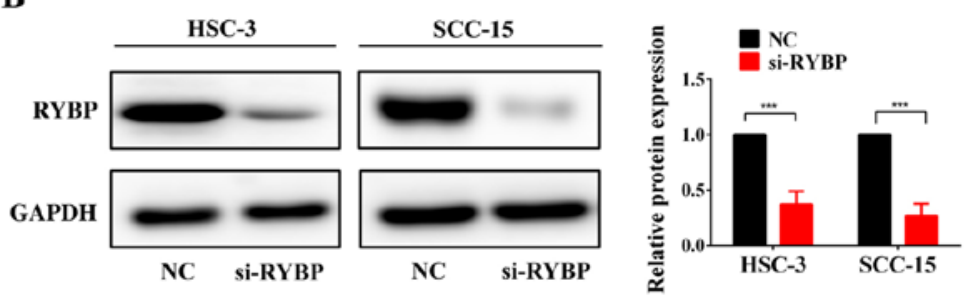

C
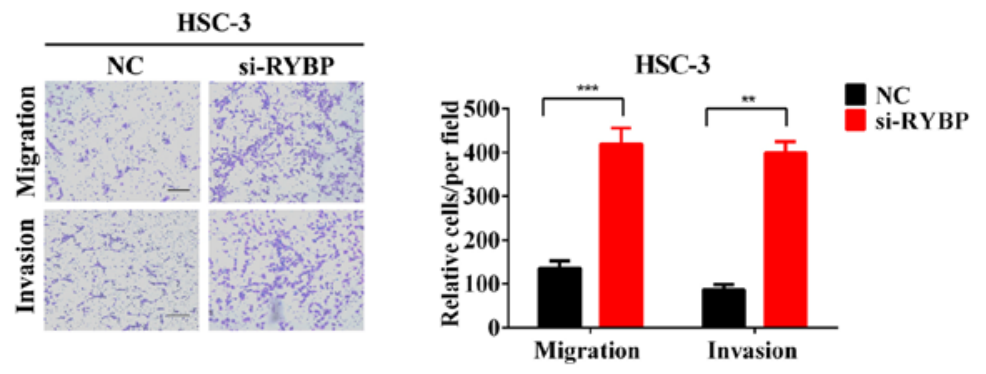

D
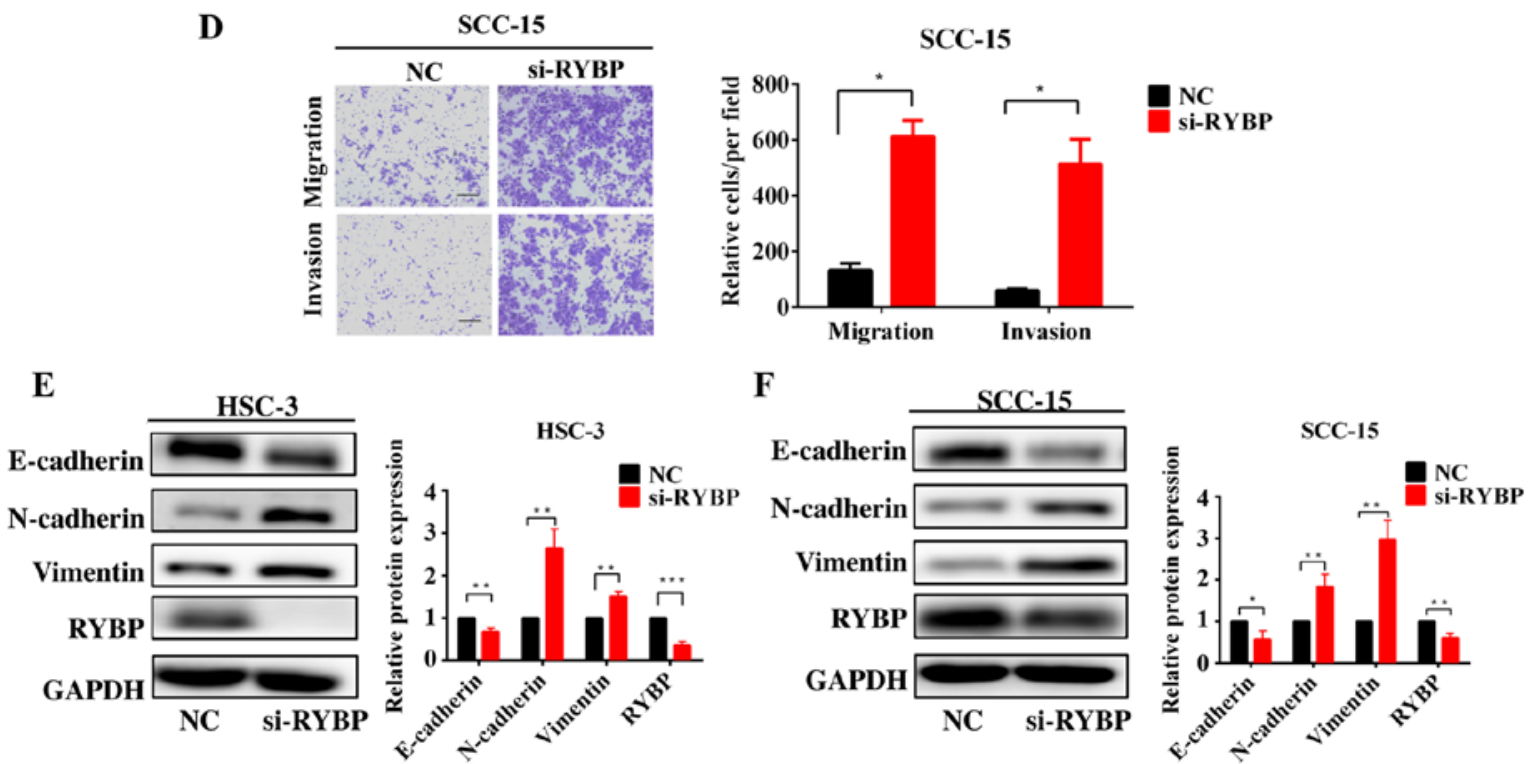

Figure 5. Knockdown of RYBP promotes HNSCC cell migration, invasion and epithelial-mesenchymal transition. (A) Expression levels of RYBP in patients with HNSCC with or without LNM were analyzed using reverse transcription-quantitative PCR. (B) Western blotting of the protein expression levels of RYBP following the silencing of RYBP in HNSCC cells. (C and D) Effects of the silencing of RYBP on HNSCC cell invasion and migration. (E and F) Western blotting of the effect of RYBP on the expression levels of E-cadherin, N-cadherin and vimentin. GAPDH was used as the internal loading control. Scale bar, $100 \mu \mathrm{m} .{ }^{*} \mathrm{P}<0.05,{ }^{* *} \mathrm{P}<0.01,{ }^{* * *} \mathrm{P}<0.001$. RYBP, RING1 and YY1 binding protein; HNSCC, head and neck squamous cell carcinoma; LNM, lymph node metastasis; si-, small interfering RNA; NC, negative control.

control siRNA (Fig. 5C and D). Furthermore, the knockdown of RYBP upregulated the expression levels of N-cadherin and vimentin, and downregulated E-cadherin expression levels in HSC-3 and SCC-15 cells (Fig. 5E and F).

Knockdown of $R Y B P$ restores miR-411-5p-induced increases in HNSCC cell migration and invasion. To determine whether the knockdown of RYBP expression was able to rescue HNSCC cell migration and invasion in the anti-miR-411-5p HNSCC cell line, si-RYBP was transfected into the anti-miR-411-5p HNSCC cell line. The results of the Transwell assays revealed that the genetic silencing of RYBP in the anti-miR-411-5p HNSCC cell line increased cell migration and invasion compared with the NC group (Fig. 6A and B). Moreover, the silencing of RYBP rescued the miR-411-5p knockdown-induced downregulation of the expression levels of mesenchymal markers and upregulation of the expression levels of epithelial markers (Fig. 6C and D). Taken together, these results indicated that miR-411-5p may regulate HNSCC cell invasion, migration and EMT by targeting RYBP.

\section{Discussion}

Metastasis is a major obstacle for the effective treatment of HNSCC (28). Previously, it has emerged that miRNAs play important roles in cancer initiation, development, drug resistance and metastasis $(5,10)$. Previous studies have demonstrated that miR-411-5p exhibits both tumor suppressive and oncogenic properties in different types of cancer (29-31). However, to the best of our knowledge, few studies have focused on the association between miR-411-5p expression levels and metastasis in HNSCC. The present study first used bioinformatics analysis to analyze the expression levels of miR-411-5p in patients with or without LNM. The results 
A

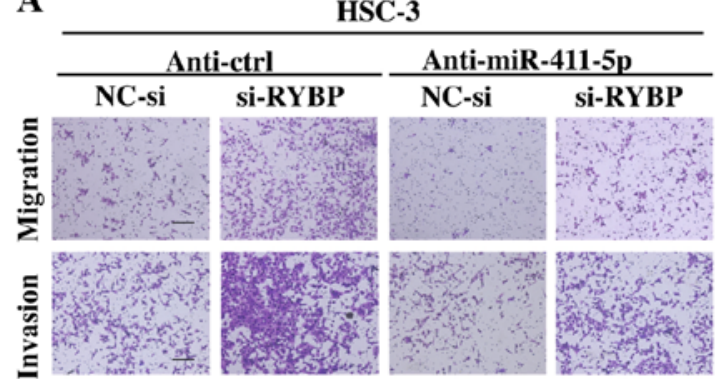

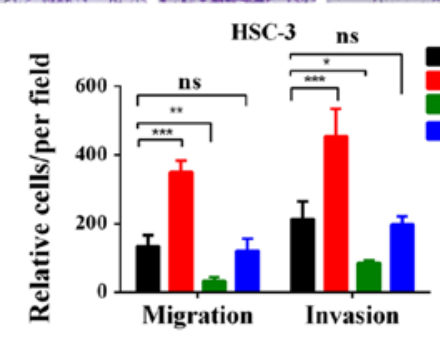

C

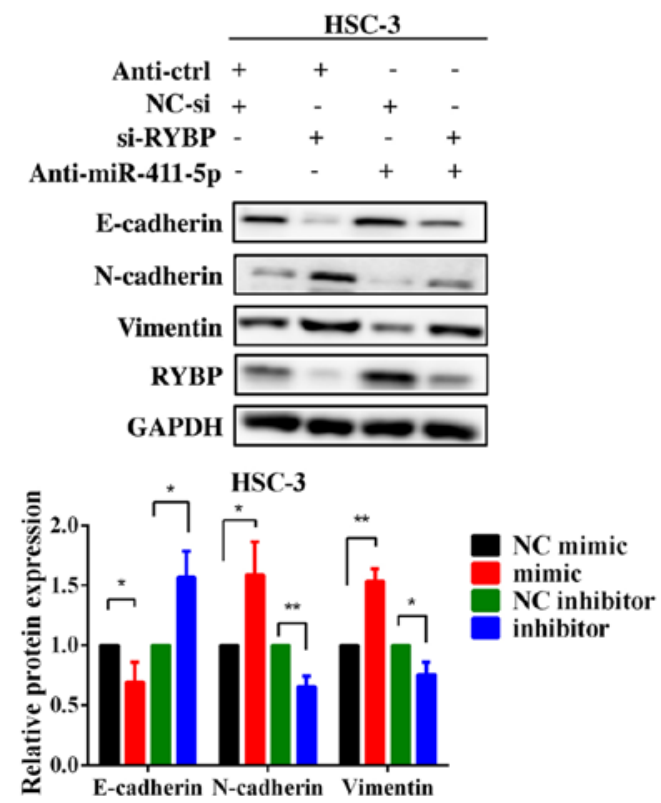

B
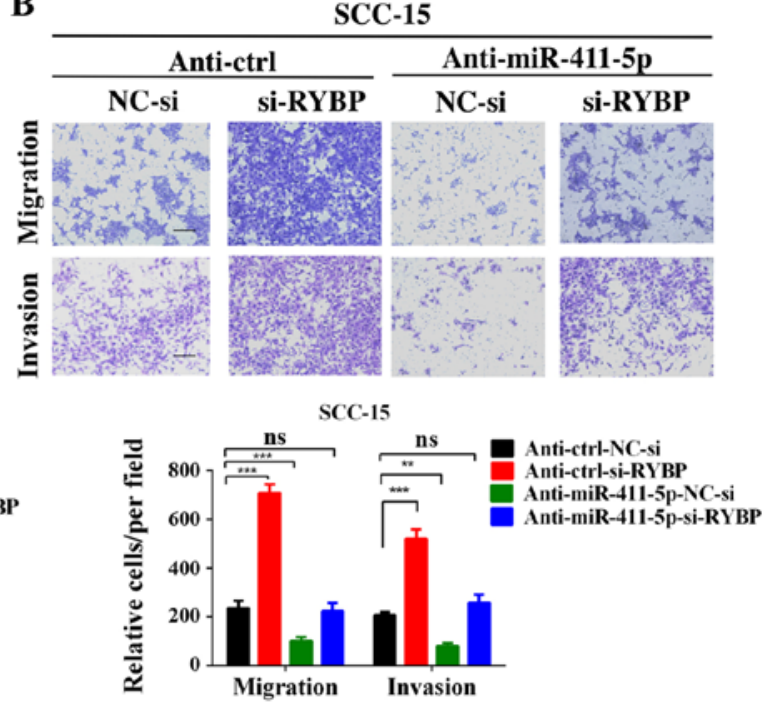

D
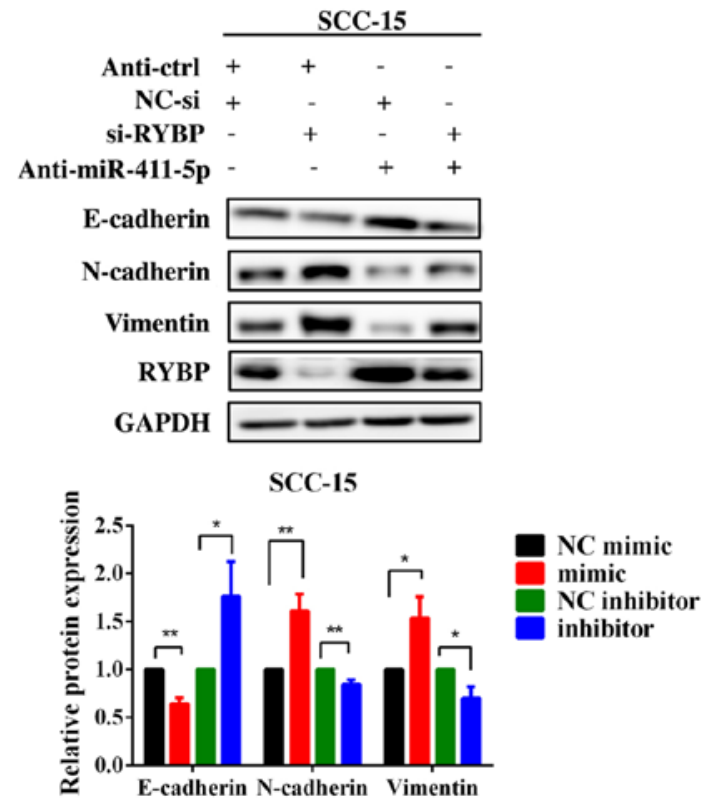

Figure 6. RYBP, as a direct target, may be responsible for miR-411-5p-induced head and neck squamous cell carcinoma cell invasion, migration and epithelial-mesenchymal transition. (A and B) Transwell assays were performed following the co-transfection of HSC-3 and SCC-15 cells with the miR-411-5p inhibitor and si-RYBP. (C and D) Western blotting of E-cadherin, N-cadherin and vimentin expression levels following the co-transfection of HSC-3 and SCC-15 cells with the miR-411-5p inhibitor and si-RYBP. GAPDH was used as the internal loading control. Scale bar, $100 \mu \mathrm{m} .{ }^{*} \mathrm{P}<0.05,{ }^{* * *} \mathrm{P}<0.01,{ }^{* * * *} \mathrm{P}<0.001$. RYBP, RING1 and YY1 binding protein; miR, microRNA; si-, small interfering RNA; ns, not significant; NC, negative control.

revealed that miR-411-5p expression levels were upregulated in patients with HNSCC with LNM. Similarly, data obtained from patient tissues further validated that miR-411-5p expression levels were upregulated in patients with HNSCC with LNM. Based on these observations, it was hypothesized that miR-411-5p may be associated with the aggressiveness and metastasis of HNSCC. Consistent with this hypothesis, the present results demonstrated that the upregulated expression levels of miR-411-5p were positively associated with the metastatic potential of HNSCC, and therefore, may serve as a potential indicator of poor prognosis in patients with HNSCC.

To investigate the effect of miR-411-5p on HNSCC migration and invasion in the present study, miR-411-5p was overexpressed and knocked down. miR-411-5p was discovered to significantly promote HNSCC cell migration and invasion, which was consistent with the reported oncogenic role of
miR-411-5p in NSCLC (17). However, miR-411-5p has been reported to inhibit proliferation and migration in bladder and gastric cancer $(32,33)$, which suggests that miR-411-5p may have opposing roles in different types of cancer and the mechanism requires further investigation. In addition, to further determine the effects on cell proliferation, CCK- 8 assays were also performed in the current study. However, the results revealed that the changes in the expression levels of miR-411-5p did not affect the levels of cell proliferation. Therefore, it was suggested that miR-411-5p may have an oncogenic role that impacts HNSCC metastasis rather than cell proliferation.

EMT serves an important role in the progression of tumor metastasis, but was first described as a feature of embryogenesis in the 1980s (34). EMT is a biological process in which epithelial cells acquire a mesenchymal phenotype or fibroblast-like properties, with an increased invasive potential (35). miRNAs 
are well-studied in the context of EMT plasticity (36). For example, Lin et al (37) reported that the downregulation of miR-639 expression levels was associated with TGF- $\beta$-induced EMT in tongue oral squamous cell carcinoma (OSCC), which may represent a possible target for the treatment of OSCC metastasis. Another previous study reported that the miR-200 family suppressed the EMT process and the metastatic ability of tongue OSCC cells (38). The findings of the present study demonstrated that miR-411-5p promoted EMT, indicating that miR-411-5p may affect the metastatic ability of HNSCC by promoting EMT.

miRNAs are known to regulate gene expression by binding to the $3^{\prime}$-UTR of target mRNAs and either inhibiting their translation or inducing the degradation of the mRNA (4). To further understand the mechanism of action of miR-411-5p in HNSCC, bioinformatics analysis was performed. The results revealed that miR-411-5p bound to the 3'-UTR of RYBP. Dual-luciferase reporter assays validated that RYBP was a direct downstream target of miR-411-5p. Further analysis confirmed that the overexpression of miR-411-5p downregulated RYBP expression levels, whereas the knockdown of miR-411-5p upregulated RYBP expression levels at both the mRNA and protein levels. RYBP is a member of the PcG of proteins (18). Dinglin et al (39) previously reported that RYBP inhibited lung cancer metastasis by reversing EMT. Another previous study reported that RYBP expression levels were negatively correlated with the upregulated expression levels of the EMT-related transcription factors, zinc finger E-box binding homeobox (ZEB)1 and ZEB2 (22). The present study revealed that RYBP expression levels were downregulated in patients with HNSCC with LNM. The genetic silencing of RYBP promoted the metastasis, invasion and EMT of HNSCC cells. In addition, the knockdown of RYBP expression restored the stimulatory effects of miR-411-5p on HNSCC cell migration, invasion and EMT, thus supporting the contribution of miR-411-5p-regulated RYBP in HNSCC metastasis and EMT. These results suggested that miR-411-5p may have the potential to be a prognostic marker and potential predictor of cervical LNM in HNSCC.

In conclusion, the findings of the present study suggested that the upregulated expression levels of miR-411-5p were associated with a poor prognosis in patients with HNSCC with LNM. Therefore, the miR-411-5p/RYBP axis may serve a significant role in promoting HNSCC metastasis and EMT.

\section{Acknowledgements}

Not applicable.

\section{Funding}

This study was supported by the National Nature Science Foundation of China (grant no. 81870769) and Guangdong Financial Fund for High-Caliber Hospital Construction (grant no. 174-2018-XMZC-0001-03-0125/D-05).

\section{Availability of data and materials}

All data generated or analyzed during this study are included in this published article.

\section{Authors' contributions}

$\mathrm{JX}$ and $\mathrm{BC}$ conceived and designed the experiments. CZ and HFW performed the experiments and collected important background information. MD, LHH and FP were involved in data acquisition and analysis. YH and ZNF drafted the manuscript and acquired data. JX and BC confirm the authenticity of all the raw data. All authors read and approved the final manuscript.

\section{Ethics approval and consent to participate}

The study was approved by the Ethics Committee of Sun Yat-Sen University (Guangzhou, China). Informed consent was obtained from all participants. All included patients fulfilled criteria and completed the study.

\section{Patient consent for publication}

All participants provided written informed consent.

\section{Competing interests}

The authors declare that they have no competing interests.

\section{References}

1. Leemans CR, Braakhuis BJ and Brakenhoff RH: The molecular biology of head and neck cancer. Nat Rev Cancer 11: 9-22, 2011.

2. Siegel RL, Miller KD and Jemal A: Cancer statistics 2019. CA Cancer J Clin 69: 7-34, 2019.

3. Inglehart RC, Scanlon CS and Silva NJ: Reviewing and reconsidering invasion assays in head and neck cancer. Oral Oncol 12: 1137-1143, 2014.

4. Shukla GC, Singh J and Barik S: MicroRNAs: Processing, maturation, target recognition and regulatory functions. Mol Cellular Pharmacol 3: 83-92, 2011.

5. Calin GA and Croce CM: MicroRNA signatures in human cancers. Nat Rev Cancer 6: 857-866, 2006.

6. Hui ABY, Lenarduzzi M, Krushel T, Waldron L, Pintilie M, Shi W, Perez-Ordonez B, Jurisica I, O'Sullivan B, Waldron J, et al: Comprehensive MicroRNA profiling for head and neck squamous cell carcinomas. Clin Cancer Res 16: 1129-1139, 2010.

7. Liu X, Bi L, Wang Q, Wen M, Li C, Ren Y, Jiao Q, Mao JH, Wang C, Wei G and Wang Y: MiR-1204 targets VDR to promotes epithelial-mesenchymal transition and metastasis in breast cancer. Oncogene 37: 3426-3439, 2018.

8. Shiiba M, Uzawa K and Tanzawa H: MicroRNAs in head and neck squamous cell carcinoma (HNSCC) and oral squamous cell carcinoma (OSCC). Cancers (Basel) 2: 653-669, 2010.

9. Li Y, He Q, Wen X, Hong X, Yang X, Tang X, Zhang P, Lei Y, Sun Y, Zhang J, et al: EZH2-DNMT1-mediated epigenetic silencing of miR-142-3p promotes metastasis through targeting ZEB2 in nasopharyngeal carcinoma. Cell Death Differ 26: 1089-1106, 2019.

10. Hayes J,Peruzzi PP and Lawler S: MicroRNAs in cancer: Biomarkers, functions and therapy. Trends Mol Med 20: 460-469, 2014

11. Dong Y, Zheng Y, Wang C, Ding X, Du Y, Liu L, Zhang W, Zhang W, Zhong Y, Wu Y and Song X: MiR-876-5p modulates head and neck squamous cell carcinoma metastasis and invasion by targeting vimentin. Cancer Cell Int 18: 121, 2018.

12. Shiah SG, Hsiao JR, Chang WM, Chen YW, Jin YT, Wong TY, Huang JS, Tsai ST, Hsu YM, Chou ST, et al: Downregulated miR329 and miR410 promote the proliferation and invasion of oral squamous cell carcinoma by targeting Wnt-7b. Cancer Res 74: 7560-7572, 2014.

13. de Carvalho AC, Scapulatempo-Neto C, Maia DC, Evangelista AF, Morini MA, Carvalho AL and Vettore AL: Accuracy of microRNAs as markers for the detection of neck lymph node metastases in patients with head and neck squamous cell carcinoma. BMC Med 13: 108, 2015. 
14. Nadal E, Zhong J, Lin J, Reddy RM, Ramnath N, Orringer MB, Chang AC, Beer DG and Chen G: A MicroRNA cluster at 14q32 drives aggressive lung adenocarcinoma. Clin Cancer Res 20 3107-3117, 2014

15. Zhang Y, Xu G, Liu G, Ye Y, Zhang C, Fan C, Wang H, Cai H, Xiao R, Huang Z and Luo Q: MiR-411-5p inhibits proliferation and metastasis of breast cancer cell via targeting GRB2. Biochem Bioph Res Commun 476: 607-613, 2016.

16. Zhang X, Zhang M, Cheng J, Lv Z, Wang F and Cai Z: MiR-411 functions as a tumor suppressor in renal cell cancer. Int J Biol Markers 32: e454-e460, 2017.

17. Zhang C, Wang H, Liu X, Hu Y, Ding L, Zhang X, Sun Q and Li Y: Oncogenic microRNA-411 promotes lung carcinogenesis by directly targeting suppressor genes SPRY4 and TXNIP. Oncogene 38: 1892-1904, 2019.

18. Garcia E, Marcos-Gutierrez C, del Mar Lorente M, Moreno JC and Vidal M: RYBP, a new repressor protein that interacts with components of the mammalian Polycomb complex, and with the transcription factor YY1. EMBO J 18: 3404-3418, 1999.

19. Zhao Q, Cai W, Zhang X, Tian S, Zhang J, Li H, Hou C, Ma X Chen H, Huang B and Chen D: RYBP expression is regulated by KLF4 and Sp1 and is related to hepatocellular carcinoma prognosis. J Biol Chem 292: 2143-2158, 2017.

20. Zhou H, Li J, Zhang Z, Ye R, Shao N, Cheang T and Wang S: RING1 and YY1 binding protein suppresses breast cancer growth and metastasis. Int J Oncol 49: 2442-2452, 2016.

21. Voruganti S, Xu F, Qin JJ, Guo Y, Sarkar S, Gao M, Zheng Z, Wang MH, Zhou J, Qian B, et al: RYBP predicts survival of patients with non-small cell lung cancer and regulates tumor cell growth and the response to chemotherapy. Cancer Lett 369: 386-395, 2015.

22. Zhu X, Yan M, Luo W, Liu W, Ren Y, Bei C, Tang G, Chen R and Tan S: Expression and clinical significanceof PcG-associated protein RYBP in hepatocellular carcinoma. Oncol Lett 13: 141-150, 2017.

23. Luan PB, Jia XZ and Yao J: MiR-769-5p functions as an oncogene by down-regulating RYBP expression in gastric cancer. Eur Rev Med Pharmacol Sci 24: 6699-6706, 2020.

24. Zhao G, Li Q, Wang A and Jiao J: YY1 regulates melanoma tumorigenesis through a miR-9 $\sim$ RYBP axis. J Exp Clin Cancer Res 34: 66, 2015.

25. Pérez Sayáns M, Chamorro Petronacci CM, Lorenzo Pouso AI, Padín Iruegas E, Blanco Carrión A, Suárez Peñaranda JM and García García A: Comprehensive genomic review of TCGA head and neck squamous cell carcinomas (HNSCC). J Clin Med 8 1896, 2019.

26. Livak KJ and Schmittgen TD: Analysis of relative gene expression data using real-time quantitative PCR and the 2(-Delta Delta C(T)) method. Methods 25: 402-408, 2001.

27. National Research Council (US) Committee for the Update of the Guide for the Care and Use of Laboratory Animals: Guide for the care and use of laboratory animals. 8th edition. National Academies Press (US), Washington, DC, 2011.
28. Loberg RD, Bradley DA, Tomlins SA, Chinnaiyan AM and Pienta KJ: The lethal phenotype of cancer: The molecular basis of death due to malignancy. CA Cancer J Clin 57: 225-241, 2007.

29. Xu N, Yang W, Liu Y, Yan F and Yu Z: MicroRNA-411 promoted the osteosarcoma progression by suppressing MTSS1 expression. Environ Sci Pollutr Res Int 25: 12064-12071, 2018.

30. Zhao J, Xu J and Zhang R: MicroRNA-411 inhibits malignant biological behaviours of colorectal cancer cells by directly targeting PIK3R3. Oncol Rep 39: 633-642, 2018.

31. Jin H, Sun W, Zhang Y, Yan H, Liufu H, Wang S, Chen C, Gu J, Hua X, Zhou L, et al: MicroRNA-411 downregulation enhances tumor growth by upregulating MLLT11 expression in human bladder cancer. Mol Ther Nucleic Acids 11: 312-322, 2018.

32. Liu Y, Liu T, Jin H, Yin L, Yu H and Bi J: MiR-411 suppresses the development of bladder cancer by regulating ZnT1. Onco Targets Ther 11: 8695-8704, 2018.

33. Bai TL, Liu YB and Li BH: MiR-411 inhibits gastric cancer proliferation and migration through targeting SETD6. Eur Rev Med Pharmaco 23: 3344-3350, 2019.

34. Gonzalez DM and Medici D: Signaling mechanisms of the epithelial-mesenchymal transition. Sci Signal 7: re8, 2014.

35. Lamouille S, Xu J and Derynck R: Molecular mechanisms of epithelial-mesenchymal transition. Nat Rev Mol Cell Bio 15: 178-196, 2014.

36. Domingues CSDC, Serambeque BP, Laranjo Cândido MS, Marto CMM, Veiga FJB, Sarmento Antunes Cruz Ribeiro AB, Figueiras ARR, Botelho MFR and Dourado MARF: Epithelial-mesenchymal transition and microRNAs: Challenges and future perspectives in oral cancer. Head Neck 40: 2304-2313, 2018.

37. Lin Z, Sun L, Chen W, Liu B, Wang Y, Fan S, Li Y and Li J: MiR-639 regulates transforming growth factor beta-induced epithelial-mesenchymal transition in human tongue cancer cells by targeting FOXC1. Cancer Sci 105: 1288-1298, 2014.

38. Tamagawa S, Beder LB, Hotomi M, Gunduz M, Yata K, Grenman R and Yamanaka N: Role of miR-200c/miR-141 in the regulation of epithelial-mesenchymal transition and migration in head and neck squamous cell carcinoma. Int J Mol Med 33: 879-886, 2014.

39. Dinglin X, Ding L, Li Q, Liu Y, Zhang J and Yao H: RYBP inhibits progression and metastasis of lung cancer by suppressing EGFR signaling and epithelial-mesenchymal transition. Transl Oncol 10: 280-287, 2017.

This work is licensed under a Creative Commons Attribution-NonCommercial-NoDerivatives 4.0 International (CC BY-NC-ND 4.0) License. 